\title{
FACTORES ASOCIADOS AL ACCESO DE MÉTODOS DE PLANIFICACIÓN FAMILIAR MODERNOS EN MUJERES EN EDAD FÉRTIL INMIGRANTES VENEZOLANAS EN PERÚ. ENPOVE 2018
}

\begin{abstract}
Jorge Segundo-Paredes ${ }^{1, a}$, Marcos Espinola-Sánchez ${ }^{1, b}$, Claudia Saldaña-Diaz $^{1, c}$, Joyce Desposorio-Robles ${ }^{3, a}$, Jacqueline Mejía-Veramendi ${ }^{4, c}$, Augusto Racchumí-Vela ${ }^{1, d}$, Pedro Arango-Ochante ${ }^{2, \mathrm{e}}$, Carlos Gonzales-Medina ${ }^{4, e}$

\section{RESUMEN}

Objetivo. Estimar la prevalencia y determinar los factores asociados al acceso de métodos de planificación familiar modernos (MPFM) en mujeres en edad fértil inmigrantes venezolanas en Perú. Materiales y métodos. Estudio observacional, transversal y analítico de la Encuesta Dirigida a la Población Venezolana que Reside en el País (ENPOVE) 2018. Se calculó la proporción de mujeres en edad fértil inmigrantes venezolanas con acceso a MPFM. Se estimaron razones de prevalencia (RP) crudas y ajustadas (RPa) del acceso a MPFM, usando modelos lineales generalizados. Resultados. Se incluyeron 3378 mujeres en edad fértil inmigrantes venezolanas. El 78,45\% no tuvo acceso a MMPF. Estar en la etapa de vida joven (RPa:1.07; IC:1.05-1.14), ser casada (RPa:1.09; IC:1.03-1.14), conviviente (RPc:1.11; IC:1.06-1.16), residir en la ciudad de Trujillo (RPa:1.21; IC:1.13-1.29) se asociaron a una mayor probabilidad de acceder de MPFM. Conclusiones. Tres de cada diez mujeres en edad fértil inmigrantes venezolanas en Perú tiene acceso a MPFM. Ser joven, casada, conviviente, y residir en la ciudad de Trujillo incrementa la probabilidad de acceder a MMPF inmigrantes venezolanas en el Perú. Los servicios de salud sexual y reproductiva deben estar priorizados a aquellas a ciudades y etapas de vida con mayores barreras en el acceso a MPFM.
\end{abstract}

Palabras claves: Migrantes, planificación familiar, salud sexual y reproductiva mujeres, Venezuela, perú (Fuente: DeCS BIREME)

\section{FACTORS ASSOCIATED WITH THE ACCESS OF MODERN FAMILY PLANNING METHODS IN WOMEN OF AGE VENEZUELAN IMMIGRANTS IN PERU. ENPOVE 2018}

\begin{abstract}
Objective. Estimate the prevalence and determine the factors associated with access to modern family planning methods (MPFM) in women of childbearing age Venezuelan immigrants in Peru. Materials and methods. Observational, crosssectional and analytical study of the Survey Directed to the Venezuelan Population Residing in the Country (ENPOVE) 2018. The proportion of women of fertile age Venezuelan immigrants with access to MPFM was calculated. Crude and adjusted prevalence ratios (RP) of MPFM access were estimated, using generalized linear models. Results. 3,378 Venezuelan immigrant women of childbearing age were included. $78.45 \%$ did not have access to MMPF. Being in the young stage of life (RPa: 1.07; IC: 1.05-1.14), being married (RPa: 1.09; IC: 1.03-1.14), living together (RPc: 1.11; IC: 1.06-1.16), residing in the city de Trujillo (RPa: 1.21; Cl: 1.13-1.29) were associated with a higher probability of accessing MPFM. Conclusions. Three out of ten women of childbearing age Venezuelan immigrants in Peru have access to MPFM. Being young, married, living together, and residing in the city of Trujillo increases the probability of accessing Venezuelan immigrants to Peru in MMPF. Sexual and reproductive health services should be prioritized to those to cities and life stages with greater barriers in accessing MPFM.
\end{abstract}

Keywords: Transients and migrants, family planing, sexual an reproductive health, women, Venezuela, Peru (Source: MeSH NLM)

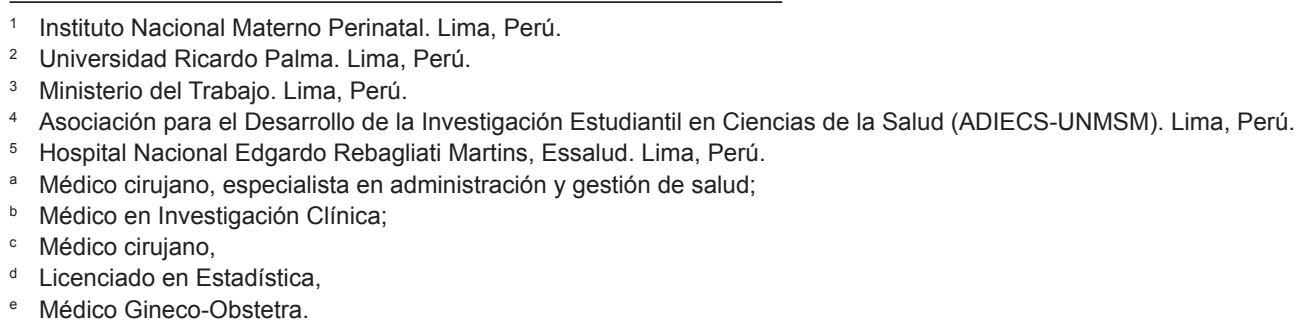

Citar como: Segundo-Paredes J, Espinola-Sánchez M, Saldaña-Díaz C, Desposorio-Robles J, Mejía-Veramendi J, Racchumí-Vela A, Arango-Ochante P, Gonzales-Medina C. Factores asociados al acceso de métodos de planificación familiar modernos en mujeres en edad fértil inmigrantes venezolanas en Perú. ENPOVE 218. Rev Peru Investig Matern Perinat 2020; 9(1): 39-44. DOI https://doi.org/10.33421/inmp.2020188

Recibido: 15-02-20 Aprobado: 12-03-20 


\section{INTRODUCCIÓN}

La inmigración, voluntaria o forzada, genera un desafío que puede contribuir a la marginación social, problemas de acceso a servicios de salud, con consecuencias adversas a la salud física y mental. El fenómeno migratorio a nivel mundial sigue un patrón de crecimiento en estos últimos años, siendo los principales factores los de índole político, económico o bélico ${ }^{1}$. En Latinoamérica y el caribe, destaca el fenómeno migratorio de la población venezolana, siendo cuatro millones los que abandonaron su país según la ONU y la OIM a mediados del 2019. Los países latinoamericanos que acogen a la gran mayoría de venezolanos son Colombia (1,3 millones); Perú (768,000); Chile (288,000); Ecuador (263,000); Argentina (130,000); Brasil $(168,000)$; entre otros ${ }^{2}$.

La Conferencia Internacional sobre Población y Desarrollo (CIPD) de 1994, celebrada en El Cairo, reconoció a los derechos sexuales y reproductivos como derechos humanos fundamentales para el desarrollo, incluyendo la planificación de la familiar y la salud sexual como parte de la salud integral de las personas y de lucha por el desarrollo económico y social. Por tal motivo, es deber de los Estados garantizar que todos puedan tener, desde su nacimiento, las mejores condiciones que permitan el logro de personas saludables. Es decir, tener acceso a la planificación familiar es un derecho de todos, más aún en personas con pobreza y/o vulnerabilidad, tales como los inmigrantes ${ }^{3-5}$.

No obstante, siguen existiendo significativas brechas en la cobertura y al acceso de servicios de salud sexual y reproductiva, en el caso de las inmigrantes, presentan barreras para el acceso efectivo a servicios formales de salud por falta de documentación, su ubicación geográfica o su poder adquisitivo ${ }^{6}$. A la fecha, hay investigación limitada que describa el estado de salud de los inmigrantes venezolanos y su acceso a métodos de planificación familiar. La OMS, pone en evidencia la necesidad insatisfecha en dicha prestación, mostrando cifras de unos 214 millones de mujeres en edad fertil que desean posponer o detener la procreación pero no utilizan ningún método anticonceptivo moderno ${ }^{7}$. Esta falta de equidad está impulsada por un aumento de la población y una escasez de servicios de planificación familiar. En África, el $24.2 \%$ de las mujeres en edad fecunda tienen una necesidad insatisfecha de anticonceptivos modernos. En Asia y América Latina y el Caribe - regiones donde la prevalencia del uso de anticonceptivos es relativamente alta-, las necesidades insatisfechas representan un $10,2 \%$ y un $10,7 \%$, respectivamente ${ }^{8}$. La OMS, recomienda el uso de métodos de planificación familiar modernos tales como; anticonceptivos orales, dispositivos intrauterinos, condones masculinos y femeninos, progesterona en inyectables, vasectomía, ligadura de trompas, parches anticonceptivos combinados, entre otros; por su mayor eficacia demostrada ${ }^{9,10}$. En ese sentido, el objetivo del presente estudio fue estimar la prevalencia y determinar los factores asociados al acceso de métodos de planificación familiar (MPFM) modernos en mujeres en edad fértil inmigrantes venezolanas en Perú.

\section{MATERIALES Y MÉTODOS}

Diseño y población de estudio. El estudio es de tipo transversal analítico de bases secundarias orientado a la Encuesta Dirigida a la Población Venezolana que Reside en el País (ENPOVE), el cual fue realizado por el Instituto Nacional de Estadística e Informática (INEI) en el periodo del 19 de noviembre al 31 de diciembre 2018. La encuesta tiene una cobertura en el área urbana de seis ciudades de capitales departamentales; Tumbes, La Libertad, Arequipa, Cusco, Lima Metropolitana y la Provincia constitucional del Callao.

El tamaño total de la muestra fue de 3611 viviendas con población venezolana; el registro de la información se realizó en forma directa a cada miembro del hogar mayor de 12 años de edad; y para los casos de personas menores de 12 años, la información fue proporcionada por el jefe del hogar o algún informante calificado. El tipo de muestreo empleado fue el de tipo probabilístico, estratificada e independiente en cada ciudad/ cono de estudio; dicha muestra fue diseñada para un nivel de inferencia en cada ciudad de estudio.

Análisis estadístico. Las medidas descriptivas utilizadas para el resumen de los datos, fueron mediante frecuencias absolutas y frecuencias relativas ponderadas junto a sus intervalos de confianza a un 95\%; para el análisis bivariado se utilizó prueba de independencia mediante el estadístico chi cuadrado con un nivel de significancia estadística del 0.05 (punto crítico para la decisión de rechazar la hipótesis nula) con el objetivo de identificar aquellas características que se encuentran asociadas a la variable de interés (uso de métodos de planificación familiar modernos); después de identificar asociación $(p<0.05)$ se procedió a cuantificar la fuerza de asociación mediante Razones de Prevalencia (RP) crudos y ajustados haciendo uso del análisis multivariado con los modelos lineales generalizados (GLM) de la familia de Poisson utilizando la función de enlace de tipo log. Finalmente se realizó un análisis de correspondencias múltiple el cual se utiliza a menudo para la representación de datos que pueden presentarse en forma de tablas de contingencia, el cual busca identificar la relación entre los niveles de categorías de las variables en estudio. El procesamiento de los datos fue realizado en el software estadístico Stata versión 15 y en el software libre $\mathrm{R}$ versión 3.6; para todas las estimaciones realizadas se consideró la especificación de diseño muestral complejo con factor de expansión de la ENPOVE. 


\section{RESULTADOS}

Las unidades de análisis fueron en total 3378 mujeres en edad fértil (MEF) inmigrantes venezolanas (con una población expandida de 229573 mujeres), de las cuales se estima que el $22.22 \%$ son jóvenes (18 a 29 años) y usan algún MPFM. Las MEF inmigrantes venezolanas convivientes que si utilizan algún MPFM es representado por una estimación del $25.41 \%$; del total de MEF inmigrantes venezolanas con educación superior técnico el $22.10 \%$ usan algún MPFM; mientras que del total de MEF inmigrantes venezolanas con un nivel de educación superior (licenciatura, maestría y doctorado) el $21.29 \%$ si usan algún MPFM.

En relación a la cobertura de salud de las MEF inmigrantes, se estima que el $27.33 \%$ de las MEF inmigrantes venezolanas que tienen SIS, usan algún MPFM, mientras que las MEF inmigrantes venezolanas que se encuentran afiliadas a ESSALUD el $21.18 \%$, usan algún MPFM. Por otro lado, las MEF inmigrantes venezolanas que hacen uso de los servicios de salud privados, el $28.58 \%$ usan MPFM y finalmente la ciudad que tienen una mayor proporción de MEF inmigrantes venezolanas que usan algún método moderno es Trujillo teniendo un estimador puntual del $46.25 \%$ y en Lima Metropolitana del $20.27 \%$.

En cuanto al análisis bivariado, se encontró que las variables: edad, estado civil y la ciudad de residencia, tienen una relación significativa con el uso de MPFM.

Se realizó las especificaciones muestrales de la ENPOVE 2018.

Se incluyó el factor de expansión para el cálculo de los porcentajes.

Los valores $p$ se obtuvieron mediante la prueba de chicuadrado de independencia.

Las medidas de fuerza de asociación entre las características que resultaron ser significativas en el análisis bivariado, se modelaron en un análisis multivariado encontrándose los siguientes resultados: ser joven tiene un $7 \%(R P=1.07 ; 1.05-1.14)$ de mayor probabilidad para usar MPFM en comparación a los adolescentes; tener un estado civil de conviviente, casado y otros (entre separados y viudos), tiene un $11 \%(R P=1.11 ; 10.6-1.16), 9 \%(R P=1.09 ; 10.3-1.14)$

Tabla 1. Características de MEF inmigrantes venezolanas de 15 a 49 años venezolanas según el uso de MPFM. ENPOVE 2018.

\begin{tabular}{|c|c|c|c|c|c|c|c|c|}
\hline & & & Uso & le método plani & ción fa & iliar mod & erno & \\
\hline inm & $\begin{array}{l}\text { aracterísticas MEF } \\
\text { igrantes venezolanas }\end{array}$ & & & & & No & & $\mathbf{p}$ \\
\hline & & $\mathbf{n}$ & Porcer & taje e IC al $95 \%$ & $\mathbf{n}$ & Porcen & taje e IC al $95 \%$ & \\
\hline & Adolescente & 10 & 9,03 & $(4,31-17,96)$ & 78 & 90,97 & $(82,04-95,69)$ & \\
\hline Edad & Joven & 436 & 22,22 & $(18,26-26,76)$ & 1441 & 77,78 & $(73,24-81,74)$ & 0,01 \\
\hline & Adulto & 282 & 18,41 & $(14,26-23,43)$ & 1131 & 81,59 & $(76,57-85,74)$ & \\
\hline & Soltero & 174 & 12,69 & $(9,46-16,81)$ & 1016 & 87,31 & $(83,19-90,54)$ & \\
\hline & Conviviente & 368 & 25,41 & $(20,35-31,22)$ & 1029 & 74,59 & $(68,78-79,65)$ & \\
\hline Estado cIVII & Casado & 156 & 21,45 & $(16,85-26,91)$ & 482 & 78,55 & $(73,09-83,15)$ & 0,00 \\
\hline & Otros & 30 & 27,42 & $(14,70-45,31)$ & 123 & 72,58 & $(54,69-85,29)$ & \\
\hline & Sin educación superior & 250 & 17,86 & $(13,76-22,85)$ & 972 & 82,14 & $(77,15-86,24)$ & \\
\hline $\begin{array}{l}\text { Nivel de } \\
\text { educación }\end{array}$ & Superior técnico & 137 & 22,10 & $(16,77-28,54)$ & 488 & 77,90 & $(71,46-83,23)$ & 0,27 \\
\hline & Superior Universitario & 341 & 21,29 & $(17,02-26,30)$ & 1190 & 78,71 & $(73,70-82,98)$ & \\
\hline & ESSALUD & 19 & 21,18 & $(11,96-34,71)$ & 61 & 78,82 & $(65,29-88,04)$ & \\
\hline Afiliado a seguro & Seguro privado & 5 & 13,71 & $(4,10-37,11)$ & 29 & 86,29 & $(62,89-95,90)$ & 040 \\
\hline de salud & SIS & 33 & 27,53 & $(17,76-40,06)$ & 115 & 72,47 & $(59,93-82,24)$ & 0,40 \\
\hline & Sin seguro & 671 & 19,95 & $(16,20-24,32)$ & 2445 & 80,05 & $(75,68-83,80)$ & \\
\hline & MINSA & 65 & 19,69 & $(13,43-27,92)$ & 189 & 80,31 & $(72,08-86,57)$ & \\
\hline & EsSalud & 6 & 17,89 & $(5,36-45,80)$ & 22 & 82,11 & $(54,20-94,68)$ & \\
\hline $\begin{array}{l}\text { Uso de servicios } \\
\text { de salud }\end{array}$ & Privado & 19 & 28,58 & $(14,85-47,86)$ & 69 & 71,42 & $(52,14-85,15)$ & 0,07 \\
\hline & Servicios Informales & 247 & 20,88 & $(16,29-26,35)$ & 788 & 79,12 & $(73,65-83,71)$ & \\
\hline & No busco & 58 & 11,22 & $(6,99-17,51)$ & 265 & 88,78 & $(82,49-93,00)$ & \\
\hline & Lima Metropolitana & 332 & 20,27 & $(16,41-24,75)$ & 1301 & 79,73 & $(75,25-83,59)$ & \\
\hline & Callao & 56 & 13,65 & $(8,77-20,65)$ & 332 & 86,35 & $(79,35-91,23)$ & \\
\hline Ciudad de & Arequipa & 73 & 14,37 & $(9,62-20,92)$ & 418 & 85,63 & $(79,08-90,38)$ & \\
\hline Residencia & Cusco & 39 & 16,36 & $(9,72-26,22)$ & 178 & 83,64 & $(73,78-90,28)$ & 0,00 \\
\hline & Trujillo & 209 & 46,25 & $(38,33-54,36)$ & 245 & 53,75 & $(45,64-61,67)$ & \\
\hline & Tumbes & 19 & 11,03 & $(5,71-20,26)$ & 176 & 88,97 & $(79,74-94,29)$ & \\
\hline
\end{tabular}


Tabla 2. Factores asociados al acceso de métodos planificación familiar modernos en MEF inmigrantes venezolanas. ENPOVE 2018.

\begin{tabular}{|c|c|c|c|c|c|c|c|}
\hline \multirow{2}{*}{\multicolumn{2}{|c|}{$\begin{array}{l}\text { Características de las MEF } \\
\text { inmigrantes venezolanas }\end{array}$}} & \multicolumn{3}{|c|}{ Análisis bivariado } & \multicolumn{3}{|c|}{ Análisis multivariado } \\
\hline & & \multicolumn{2}{|c|}{ RP e IC AL $95 \%$} & \multirow{2}{*}{$\frac{\text { Valor } \mathbf{p}}{-}$} & \multicolumn{2}{|c|}{ RP e IC AL $95 \%$} & \multirow[t]{2}{*}{ Valor $p$} \\
\hline \multirow{3}{*}{ Edad } & Adolescente & Ref & - & & Ref & & \\
\hline & Joven & 1,12 & $(1,05-1,20)$ & 0,00 & 1,07 & $(1,05-1,14)$ & 0,04 \\
\hline & Adulto & 1,09 & $(1,01-1,17)$ & 0,03 & 1,02 & $(0,95-1,10)$ & 0,53 \\
\hline \multirow{4}{*}{ Estado civil } & Soltero & Ref & - & - & Ref & - & - \\
\hline & Conviviente & 1,11 & $(1,07-1,16)$ & 0,00 & 1,11 & $(1,06-1,16)$ & 0,00 \\
\hline & Casado & 1,08 & $(1,03-1,13)$ & 0,00 & 1,09 & $(1,03-1,14)$ & 0,00 \\
\hline & Otros & 1,13 & $(0,01-1,27)$ & 0,04 & 1,14 & $(1,02-1,28)$ & 0,02 \\
\hline \multirow{3}{*}{ Nivel de educación } & Sin educación superior & Ref & - & - & - & - & - \\
\hline & Superior técnico & 1,04 & $(0,98-1,09)$ & 0,19 & - & - & - \\
\hline & Superior Universitario & 1,03 & $(0,99-1,08)$ & 0,20 & - & - & - \\
\hline \multirow{4}{*}{ Afiliado a seguro de salud } & ESSALUD & 1,01 & $(0,92-1,11)$ & 0,84 & - & - & - \\
\hline & Seguro privado & 0,95 & $(0,83-1,08)$ & 0,43 & - & - & - \\
\hline & SIS & 1,06 & $(0,97-1,17)$ & 0,21 & - & - & - \\
\hline & Sin seguro & Ref & - & - & - & - & - \\
\hline \multirow{5}{*}{ Uso de servicios de salud } & MINSA & 0,99 & $(0,92-1,07)$ & 0,79 & - & - & - \\
\hline & EsSalud & 0,98 & $(0,83-1,15)$ & 0,76 & - & - & - \\
\hline & Privado & 1,06 & $(0,93-1,22)$ & 0,37 & - & - & - \\
\hline & Servicios Informales & Ref & - & - & - & - & - \\
\hline & No busco & 0,92 & $(0,87-0,97)$ & 0,00 & - & - & - \\
\hline \multirow{6}{*}{ Ciudad de residencia } & Lima Metropolitana & Ref & - & - & Ref & - & - \\
\hline & Callao & 0,95 & $(0,89-1,01)$ & 0,07 & 0,95 & $(0,89-1,01)$ & 0,08 \\
\hline & Arequipa & 0,95 & $(0,90-1,01)$ & 0,10 & 0,95 & $(0,89-1,01)$ & 0,08 \\
\hline & Cusco & 0,97 & $(0,90-1,05)$ & 0,40 & 0,96 & $(0,89-1,04)$ & 0,30 \\
\hline & Trujillo & 1,22 & $(1,14-1,30)$ & 0,00 & 1,21 & $(1,13-1,29)$ & 0,00 \\
\hline & Tumbes & 0,92 & $(0,86-0,99)$ & 0,03 & 0,91 & $(0,85-0,98)$ & 0,01 \\
\hline
\end{tabular}

Se realizó las especificaciones muestrales de la ENPOVE 2018

Se incluyó el factor de expansión para el cálculo de las razones de prevalencias crudas y ajustadas

Los valore $\mathrm{p}$ se obtuvieron mediante el estadístico de Wald

y $14 \%(\mathrm{RP}=1.14 ; 1.02-1.28)$ respectivamente más probabilidades de usar un MPFM en comparación con las solteras.

Las MEF inmigrantes venezolanas que viven en la ciudad de Trujillo tienen un $21 \%$ ( $R P=1.21 ; 1.13-1.29$ ) más de probabilidad en usar un MPFM en comparación a las MEF inmigrantes venezolanas que viven en la ciudad de Lima y las MEF inmigrantes venezolanas que viven en Tumbes tienen 9\% ( $R P=0.91 ; 0.85$ - 0.98) menos de probabilidad para usar un MPFM en comparación a las que viven en Lima Metropolitana.

Con la finalidad de poder encontrar un patrón de asociación entre los niveles de las características de las MEF inmigrantes venezolanas y su preferencia de uso de MPFM, se encontró que aquellas que residen en todas las ciudades a excepción de Trujillo, son las que presentan una mayor proporción de personas que no acceden algún MPFM y junto a la característica de que dichas mujeres son de estado civil solteras; así mismo se evidencia que las MEF que viven en Trujillo usan MPFM y que en su mayoría son de estado civil de convivientes e incluso jóvenes.

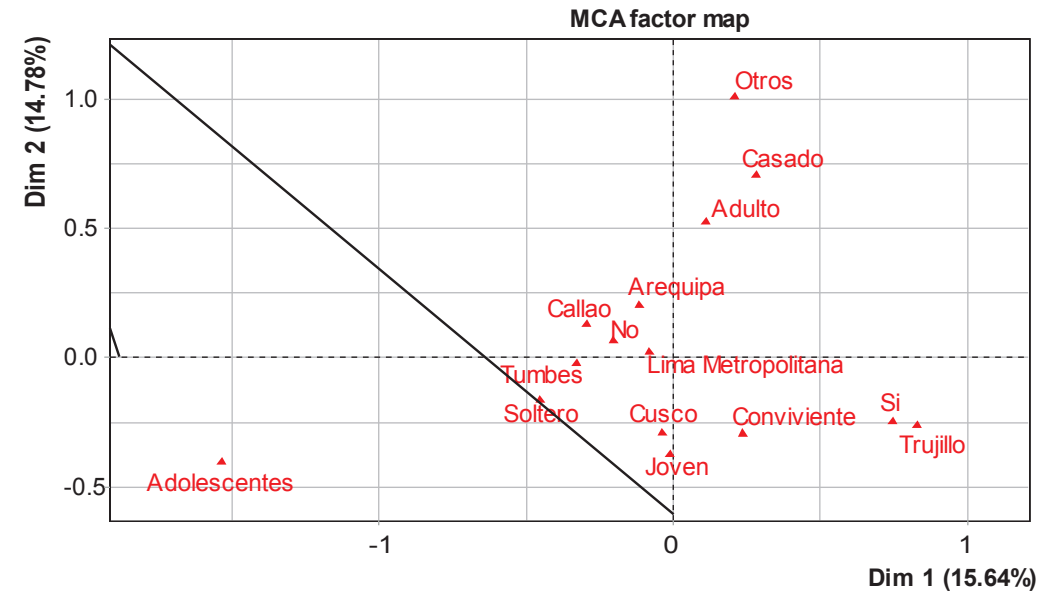

Gráfico 1. Análisis de correspondencias múltiples de las características asociadas a la utilización de métodos de planificación familiar modernos en MEF inmigrantes venezolanas. ENPOVE 2018. 


\section{DISCUSIÓN}

Los inmigrantes en su condición de población vulnerable y además por la segmentación y fragmentación del sistema de salud peruano tienen barreras en el acceso de servicios de salud. ${ }^{11}$ La dificultad es muy similar en la población peruana según los sugieren estudios previos. ${ }^{12,13}$ pese a haber presentado algún síntoma, malestar, enfermedad, recaída de enfermedad crónica o accidente durante el último mes, no acudieron a los servicios de salud. Se analizaron 35036 participantes; la prevalencia de NUSFPS fue de 53,9\% (IC95\%:52,9-54,8 La utilización de servicios formales de salud es de una baja prevalencia, y la salud sexual y reproductiva no es la excepción. El estudio incluyo a mujeres en edad fértil inmigrantes venezolanas, los resultados obtenidos sugieren una baja utilización de métodos modernos de planificación familiar. Las inmigrantes jóvenes, casadas y convivientes tienen más probabilidades de acceder a métodos de planificación familiar modernos en comparación con las solteras. Las inmigrantes que residen en la ciudad de Trujillo tienen más probabilidades de acceder a métodos de planificación familiar en comparación con las que residen en Lima. Los hallazgos encontrados nos indican que una importante proporción de mujeres en edad fértil no están recibiendo acceso a servicios de planificación familiar, exponiéndose a infecciones de transmisión sexual y a embarazos no deseados, esto impactaría definitivamente en su condición socio-económica y en su estado de salud ${ }^{14-16}$.

La población de mujeres en edad fértil en etapa de vida adulta tiene menos probabilidad de acceder a servicios de salud sexual y reproductiva. Esto podría explicarse ya que según la encuesta ENPOVE realizado por el INEI, se evidencio que las inmigrantes venezolanas trabajan largas jornadas horarias superando las ocho horas diarias, sin beneficios por carecer en su gran mayoría de la documentación respectiva ${ }^{17}$. A esto, se le suma, la sobrecarga actual de los servicios de salud, todo ello, contribuiría a que existan más barreras con los servicios de planificación familiar.

El presente estudio cuenta con algunas consideraciones que podrían limitar la interpretación de los hallazgos encontrados y es importante tenerlas en consideración. En primer lugar, es un estudio de análisis secundario de base de datos, por tal motivo, los datos recolectados dependerán del correcto levantamiento de información del personal de campo del estudio original, además podría estar presente un sesgo de memoria. En segundo lugar, el tipo de diseño del estudio es transversal, por ende, no permite evaluar relación de causalidad, sólo asociaciones que podrían ser evaluadas posteriormente en estudios de tipo prospectivo y con mayores recursos. Es decir no permite evaluar la causalidad entre factores asociados al acceso de métodos de planificación familiar modernos dada la ausencia de temporalidad entre las variables. Por otro lado, el estudio tiene fortalezas, al ser un estudio basado en datos de una Encuesta Nacional por lo que, en base a la metodología diseñada para dicha encuesta, la población peruana se puede ver representada en la muestra de estudio.

En conclusión, se encontró que tres de cada diez mujeres en edad fértil inmigrantes venezolanas accede a MPFM. Ser joven, casada, conviviente, y residir en la ciudad de Trujillo incrementa la probabilidad de acceder a MMPF inmigrantes venezolanas en el Perú. Es importante analizar las buenas prácticas de las ciudades con mayor acceso para que puedan ser trasladadas a las que tengan cifras menos favorables. Los servicios de salud sexual y reproductiva son un derecho humano, ser inmigrante y vulnerable exige al sistema de salud garantizar dicha prestación, por ende, deben estar priorizados a aquellas a ciudades y etapas de vida con mayores barreras en el acceso a métodos de planificación familiar modernos.

Financiamiento: Autofinanciado.

Conflicto de interés: Los autores declaran no tener algún conflicto de intereses.

\section{REFERENCIAS BIBLIOGRÁFICAS}

1. Shishehgar S, Gholizadeh L, DiGiacomo M, Davidson PM. The impact of migration on the health status of Iranians: an integrative literature review. BMC Int Health Hum Rights. 15 de agosto de 2015;15:20.

2. Organización Internacional para las Migraciones (OIM). ACNUR - Refugiados y migrantes de Venezuela superan los cuatro millones: ACNUR y OIM [Internet]. 2019 [citado 16 de marzo de 2020]. Disponible en: https://www.acnur.org/ noticias/press/2019/6/5cfa5eb64/refugiados-y-migrantes-devenezuela-superan-los-cuatro-millones-acnur-y.html

3. Galdos Silva S. La conferencia de El Cairo y la afirmación de los derechos sexuales y reproductivos, como base para la salud sexual y reproductiva. Rev Peru Med Exp Salud Publica. julio de 2013;30(3):455-60.

4. Migración internacional e inclusión en América Latina: Análisis en los países de destino mediante encuestas de hogares | Publicación | Comisión Económica para América Latina y el Caribe [Internet]. [citado 16 de marzo de 2020]. Disponible en: https://www.cepal.org/es/publicaciones/43947-migracioninternacional-inclusion-america-latina-analisis-paises-destino

5. La planificación familiar como herramienta básica para el desarrollo [Internet]. [citado 16 de marzo de 2020]. Disponible en: $\quad$ http://www.scielo.org.pe/scielo.php?script=sci_ arttext\&pid=S1726-46342013000300016

6. Mendoza W, Miranda JJ. La inmigración venezolana en el Perú: desafíos y oportunidades desde la perspectiva de la salud. Rev Peru Med Exp Salud Pública. 23 de septiembre de 2019;36(3):497-503.

7. Organización Mundial de la Salud. Planificación familiar [Internet]. [citado 23 de marzo de 2020]. Disponible en: https://www.who.int/es/news-room/fact-sheets/detail/familyplanning-contraception

8. United Nations, Department of Economic and Social Affairs, Population Division. Trends in contraceptive use worldwide, 2015. 2015. 
9. OMS | Criterios médicos de elegibilidad para el uso de anticonceptivos [Internet]. WHO. World Health Organization; [citado 16 de marzo de 2020]. Disponible en: http://www. who.int/reproductivehealth/publications/family_planning/ExSumm-MEC-5/es/

10. OMS | Recomendaciones sobre prácticas seleccionadas para el uso de anticonceptivos [Internet]. WHO. World Health Organization; [citado 16 de marzo de 2020]. Disponible en: http://www.who.int/reproductivehealth/publications/family planning/SPR-3/es/

11. Ugarte-Ubilluz O. Desarrollo y ejercicio de los derechos en salud sexual y reproductiva. Rev Peru Med Exp Salud Publica. julio de 2013;30(3):376-8.

12. Benites-Zapata VA, Lozada-Urbano M, Urrunaga-Pastor D, Márquez-Bobadilla E, Moncada-Mapelli E, Mezones-Holguin E. Factores asociados a la no utilización de los servicios formales de prestación en salud en la población peruana: análisis de la Encuesta Nacional de Hogares (ENAHO) 2015. Rev Peru Med Exp Salud Pública. 9 de octubre de 2017;34(3):478-84

13. Hernández-Vásquez A, Vargas-Fernández R, Rojas-Roque C, Bendezu-Quispe G. Factores asociados a la no utilización de servicios de salud en inmigrantes venezolanos en Perú. Rev Peru Med Exp Salud Pública. 2019;36(4):583-91.

14. Rebolledo-Ponietsky K, Munayco CV, Mezones-Holguín E. Migration crisis in Venezuela: impact on HIV in Peru. J Travel Med [Internet]. 1 de febrero de 2019 [citado 12 de marzo de 2020];26(2). Disponible en: https://academic.oup.com/jtm/ article/26/2/tay155/5259063
15. Tamagno C. Informe Técnico: "Situación de los Migrantes Extranjeros en el Perúy suaccesoa servicios sociales, servicios de salud y de educación" [Internet]. 2015 [citado 12 de marzo de 2020]. Disponible en: https://peru.iom.int/sites/default/files/ Documentos/03112016Informe\%20FinalExtranjerosPERU OIM.pdf\#overlay-context=publicaciones

16. Abubakar I, Aldridge RW, Devakumar D, Orcutt M, Burns R, Barreto ML, et al. The UCL-Lancet Commission on Migration and Health: the health of a world on the move. The Lancet. 15 de diciembre de 2018;392(10164):2606-54.

17. INEI. Instituto Nacional de Estadistica e Informatica. Condiciones de vida de la población venezolana que reside en Perú [Internet]. 2018 [citado 12 de diciembre de 2019]. Disponible en: https://www.inei.gob.pe/media/ MenuRecursivo/publicaciones_digitales/Est/Lib1666/libro.pdf

\section{Correspondencia:}

Jorge Segundo Paredes

Dirección: Calistemo 977 Urbanización Las Palmeras Los Olivos Correo: dr.jorgesegundo@gmail.com

Teléfono: 942085418 